\title{
RADIAL EXTARCORPOREAL SHOCK WAVES: THERAPEUTIC MYOFASCIAL PROTOCOL
}

${ }^{\prime}$ PhD, Coordinator Center for Rehabilitation and Reeducation (Ce.Fi.R.R.) venue "G. d'Annunzio" University of Chieti-Pescara (Italy).

${ }^{2} M D$, Physical Medicine and Rehabilitation Specialist, Sector of Clinical Evaluation and Diagnosis of Ce.Fi.R.R. venue "G. d'Annunzio" University of Chieti-Pescara (Italy); Physiatrist for the Local Health Company ASL Chieti (Italy).

${ }^{3} M D$, Physical Medicine and Rehabilitation Specialist, Sector of Clinical Evaluation and Diagnosis of Ce.Fi.R.R. venue "G. d'Annunzio" University of Chieti-Pescara (Italy); Physiatrist for the Local Health Company ASL Foggia (Italy).

${ }^{4}$ MSc, U.O.C. Thoracic Surgery. "Santo Spirito" Hospital Pescara, ASL Pescara (Italy);

${ }^{5}$ Ce.Fi.R.R. Gemelli Molise, "Catholic University of Sacred Heart"- Rome, Campobasso professionalizing teaching center.

${ }^{6}$ Associate Professor Department of Aging Sciences, "G.d'Annunzio” University Chieti-Pescara.

KEYWORDS: Extracorporeal Shockwave Therapies, Shock Wave Therapy, Physiotherapy, Rehabilitation, Physical Medicine and Rehabilitation,

\section{ABSTRACT}

The purpose of this study is to evaluate the effectiveness of radial shock waves in myofascial upper trapezius syndrome following the global treatment scheme of key trigger points.

Materials and methods

26 patients treated with rESWT (Radial shock wave therapy) after a global postural and myofascial assessment of the patient and the identification of the key trigger points.

At the end of the treatment each subject was re-evaluated through the Numerical Rating Scale - NRS and through the examination with Electronic Baropodometer.

In this study, we compared the values of NRS and the values of postural biometrics before and after 2 sessions of $r E S W T$, and a statistically significant difference $(P<0.05)$ in all measurements emerged in the values measured with NRS.

In the static percentage load, a statistically significant reduction in the percentage load differences (right / left) (P $<0.05)$ was observed in all three evaluation moments (T0, T1, T2).

The percentage and statistically significant improvement $(P<0.05)$ were recorded at time $T 2$ in the surface of the ellipse.

Greater control of balance and greater awareness of the base of support was found in the calculation of the sway path, in particular with closed eyes.

Based on this experience, the use of radial shock waves in pathologies related to myofascial pain with postural implications would be desirable.

\section{INTRODUCTION}

ESWT (shock wave therapy) is considered as the treatment of choice in numerous somatic pathologies, with interesting short- and medium-term results. Two forms of ESWT are available: focused (fESWT) and radial (rESWT) (Gerdesmeyer et al., 2002).

Focal and radial ESWTs have several characteristics in common but diverge in the depth of penetration into the tissue, in some physical characteristics and in the technique to generate them (Ogden, 2001). However, both are single acoustic pulses with a high initial positive peak pressure between 10 and 100 $\mathrm{MPa}$ reached in less than $1 \mu$ s and are characterized by a short life cycle of approximately $10-20 \mu$ s and a wide spectrum of frequencies.

Radial shock waves have lower pressure peaks than focal shock waves (5Bar) with a longer action time. The action of the rESW develops through a pneumatic generator that pushes a diffusing projectile inside a handpiece to hit an applicator (of various types) placed in contact with the patient's skin (a projectile is fired inside a tube guide that hits a metal applicator positioned on the skin). The projectile generates pressure and mechanical-sound waves transmitted to the underlying tissues.

The therapeutic action of the rSWT develops from the skin and then from the outside to the inside, where the depth is determined by the right compromise: type of diffuser, pressure intensity, frequency of action.

The amplitude of the positive pressure is followed by a low traction amplitude lasting a few microseconds which can generate cavitation.

There are several studies in the literature that show the effects of focal and radial shock wave therapy:

- One effect is the rearrangement of endothelial cells and the basal lamina with a significant increase in growth factors, increased release of NO, TGF $\beta 1$ and nuclear cell proliferation antigen (Wang et al., 2003);

- Another effect found was the bactericidal effect on Staphyilococcus Aureus crops (Horn, 2009).

But the most important and significant effects are on the reduction of inflammation and on the neoformation of vessels and cells.

In particular, a study has shown (Kuo et al., 2009) how ESWT reduce the inflammatory response with a consequent reduction in the share of circulating 
leukocytes and free radicals of oxygen and promote the production of fibroblasts and the vascularization of damaged skin through the reduction apoptotic cells. Vetrano (Vetrano, 2011), on the other hand, demonstrated how ESWTs are able to promote the growth of tenocytes and the synthesis of collagen, while in a review of 2012 (Frairia \& Berta, 2011) the biological effects of ESWT were investigated on fibroblastic activation by stimulating the production of TGF $\beta 1$ and FGF2 in vitro.

Fibroblasts are important in myofascial pain because they represent the most mechanoresponsive cell type and play a fundamental role in the remodeling of the extracellular matrix by synthesizing and reorganizing the components of the connective tissue (Lee et al., 2011).

Myofascial pain syndrome (MPS) is a common cause of pain in clinical practice.

MPS is characterized by areas known as myofascial trigger points ( $\mathrm{TrP}$ ) associated with a hyper irritable point in a taut band of a skeletal muscle.

TrP is a very small and localized area of muscle contraction, difficult to touch, painful in compression, stretching, overload or contraction of muscle and usually has a distinct reported pain pattern (Simons, 1996).

The exact pathophysiology and etiology of $\operatorname{TrP}$ and MPS are still unknown. However, many proposed mechanisms have been studied and reported in the literature.

It has been suggested, among other hypotheses, that myofascial pain is a complex form of neuromuscular dysfunction consisting of motor and sensory abnormalities involving both the peripheral and central nervous systems (Shah et al., 2008).

The diagnosis of MPS, which occurs with 1 or more active TrPs, is usually based on the patient's subjective symptoms and on the presence of an active TrP characterized by points contracted in 1 or more palpable tense bands, a reported pain pattern, response of local contraction (LTR) and limited range of motion (ROM) (Kuan et al., 2007).

Traditional therapeutic approaches for the treatment of MPS include pharmacotherapy, physical and instrumental rehabilitation therapy and lifestyle modification.

Although recent studies have shown some degree of efficacy, it is still unclear which energy level of ESWT is most suitable for pain relief and clinical improvement in MPS (Ramon et al., 2015).

Therefore, we conducted an experimental study to evaluate the effectiveness of a new low-energy therapeutic approach with rESWT on both pain and posture in patients with myofascial upper trapezius muscle syndrome.

\section{MATERIALS AND METHODS \\ Population}

After obtaining approval from patients and following the indications of the Helsinki declaration principles, 26 subjects with an average age of 51.3 years were recruited at the outpatient clinic for musculoskeletal pain (CeFiRR, center of Physiotherapy and Rehabilitation, seat of the university "Gabriele d'Annunzio of Chieti-Pescara) from 3 November 2019 to 31 January 2020.

Therapeutic protocol
All participants signed the informed consent for the experimental procedure, which complies with the latest revision of the Helsinki Declaration and with the procedures defined by the ISO 9001 standards for "Research and Experimentation"; this procedure also protects the privacy of subjects participating in biomedical research.

The diagnosis of MPS in the upper trapezius muscle was made by specialists in physiatry and pain therapy based on the criteria of Simons (Simons, 1996).

In addition, all patients were assessed at T0 (before treatment), T1 (after the first treatment) and T2 at the end of the therapeutic cycle.

The exclusion criteria for this study included patients with serious diseases (heart disease, liver disease, neoplasms), severe psychiatric problems, systemic rheumatic diseases, neurological disorders such as cervical radiculopathy or stroke, bleeding tendency, a history of side effects to local anesthetics. , presence or suspicion of infection, patients using a pacemaker, those who have undergone drug therapy (other than acetaminophen) or anticoagulant therapy, those under the age of 19 or over 70 and pregnant or breastfeeding women, which could affect the results during the treatment or the period.

Patients were treated with low energy radial ESWT (PR 3.0, FR $10 \mathrm{~Hz}$ ).

The experimental group received 4000 impulses once a week for 2 weeks.

The rESWT treatment involved the patient's overall and myofascial postural evaluation and an identification of the key trigger points.

The key trigger point represents the somatic structure that expresses the greatest dysfunction both from a tissue point of view and from the articulation of the movement and asymmetry of the segments examined. The key trigger point can be active or latent, but still represents a somatic dysfunction (Barassi et al., 2018).

All patients had to stop pain relievers, including nonsteroidal anti-inflammatory drugs (NSAIDs), corticosteroids, antidepressants and muscle relaxants, for one week before the first ESWT treatment.

All rESWT treatments were performed by 2 health professionals with relevant experience, using a radial shock wave generator (6000 SWT Topline, BTL Industries $($, Stevenage, Hertfordshire, Gran Bretagna).

For trapezius muscle treatment, patients were asked to sit in a backless chair and lower their arm.

For the target position, the operator examined the $\operatorname{TrP}$ of the upper trapezius and applied the focus on the area (TrP) to which to induce the response of muscle contractions or of the pain reported, appropriately adjusting the position of the localized probe.

\section{Evaluation methods}

The presence of the other active or latent malfunction trigger points was objectified through:

- a biometric postural evaluation, which allowed us to highlight any load asymmetries and balance alterations (electromedical equipment, such as stabiloposturometric platform: $1.5 \mathrm{~cm}$ high platform with and with adjustable (DIASUC) board, Milletrix (C) software, DIASU(C) Corporate, Diasu Health Technologies (C, Sa.Ni Corporate S.r.l., Roma, Italy) (Aprile et al., 2013; Bellizzi et al., 2013); 
- a kinesiological and manual evaluation by the physiotherapist, which allowed us to identify and confirm ROM and tissue alterations due to load asymmetries.

- an assessment of skin impedance to specifically identify dysfunctional myofascial areas, using the ENF Physio ${ }^{\circledR}$ tool (ElectroNeuroFeedback) capable of interacting with skin receptors and localizing dysfunctional somatic structures throughout the muscle course (Barassi et al., 2019);

- When the MPS was diagnosed bilaterally, the more dysfunctional side was treated.

Each patient was treated at three points on average for each hemisome, in addition to the upper trapezius muscle, and in general the key trigger points were identified in the muscles:

square of the sole of the foot, twins, piriformis, lumbar paravertebral, rhomboid, subscapular, supraspinous and pectoralis major.

At the end of the treatment, each subject was reevaluated through an examination with an electronic baropodometer (Diasu Health Technologies C), Sa.Ni Corporate S.r.l., Roma, Italy).

The posturometric examination analyzes the distribution of body weight loads on its support base and expresses important information on the podalic and center of gravity support.

This investigation, which identifies functional synergies during static, is repeated several times to identify load distribution and balance deficits between the two sides of the body.

In the study, the pressure centers of each foot are elaborated, divided into frames to highlight the hindfoot with respect to the forefoot and the numeric values of surface and load, times of constraint reactions to the ground and resulting barycentric.

The balance test, which allows to study the possible destabilizing effect induced by the perturbation of one or more sensory inputs, is carried out on the baropodometric platform with complementary stabilometric tests in bipodalic support at $30^{\circ}$ and in monopodalic, with eyes open and closed : the data obtained present the patient's oscillations on the anterior / posterior and latero / lateral planes and are used to identify oculomotor and vestibular problems, as well as problems of the proprioceptive system (Romberg index).

Pain was assessed on the NRS scale. The same evaluations were carried out fifteen days after the patients had also performed the second and last therapeutic session.

\section{RESULTS}

In this study, we compared NSR values and postural biometrics values, before and after 2 rESWT sessions. The quantitative analysis of the collected data was performed using Office Excel software (Microsoft ${ }^{\circledR}$, Redmond, Washington, USA), through which it was possible to create a graphic representation of the average variation of the values taken into consideration, through the calculation of the mean, standard deviation, percentage improvement and Student's T-Test, to evaluate its statistical significance. The results showed a significant improvement in the group under consideration both at the measurements in the first treatment and at the end of the therapeutic cycle. (Fig.1)

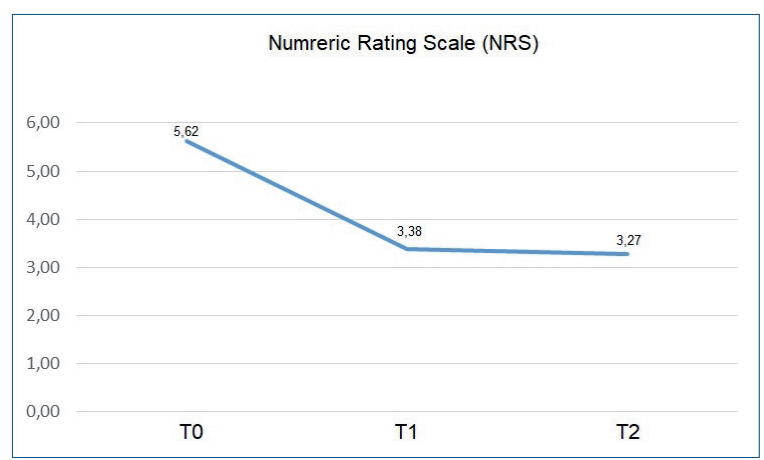

Fig. 1 - Mean variation of the NRS scale for pain evaluation.

In the values measured with NSR there is a statistically significant difference $(\mathrm{P}<0.05)$ in all the measurements, an evident sign of the reduction of pain, which has been maintained over time (Figure 1).

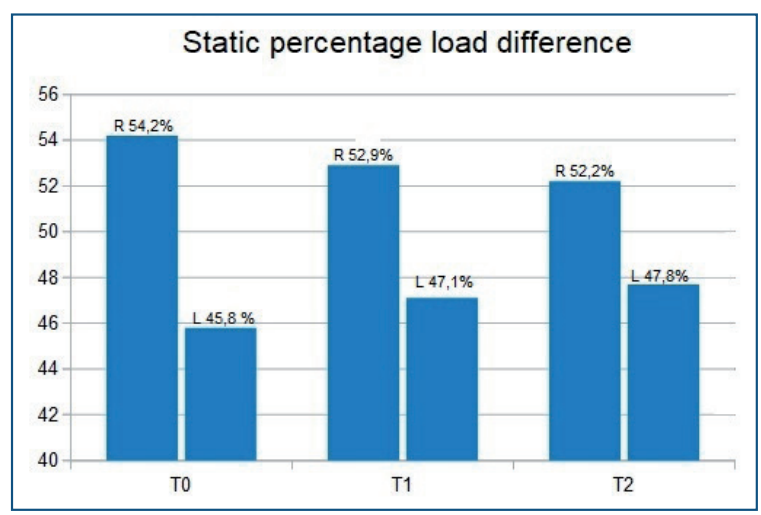

Fig. 2 - Mean static load percentage difference between right $(R)$ and left $(S)$ side of the body.

In the static percentage load assessment, a statistically significant reduction in the percentage load differences (right / left) $(\mathrm{P}<0.05)$ was observed in all three evaluation moments (T0, T1, T2) (Figure 2).

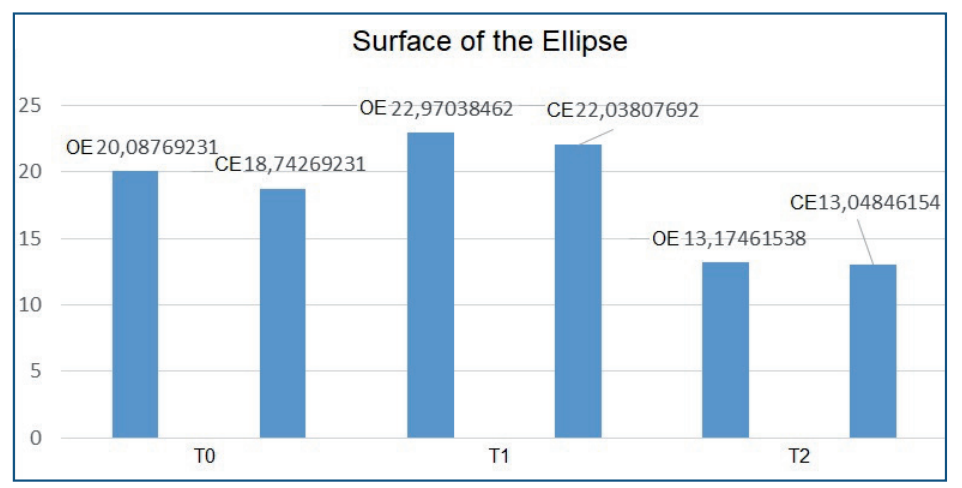

Fig. 3 - Mean surface of the Ellipse with eyes open (OE) and eyes closed (CE).

The stabilometric examination instead shows the ellipse surface: this parameter allows us to establish the impact of oscillations on the support surface (Figure 3). The percentage and statistically significant improvement were recorded at time $\mathrm{T} 2$, while at time T1 there was an immediate adaptation that had increased these values.

Greater control of balance and greater awareness of the base of support was found in the values calculated in the length of the CoP Path Lenght, in particular with the eyes closed. The CoP Path Length is the 
Lenght of the CoP Path

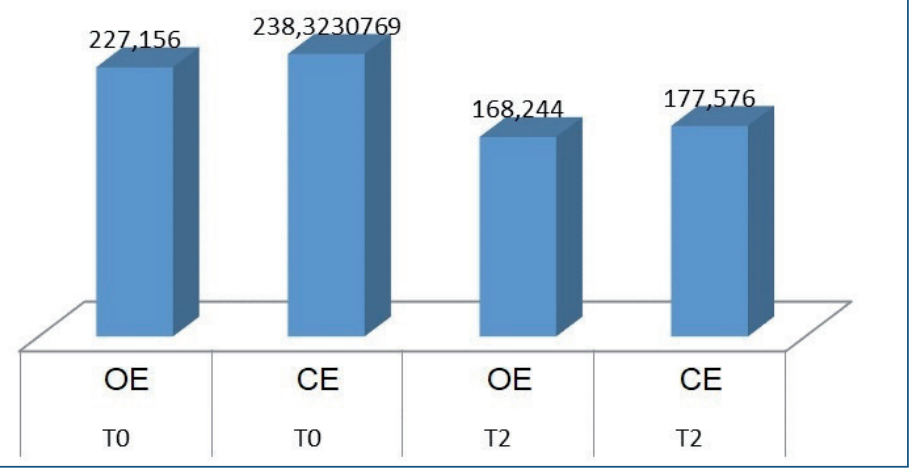

Fig. 4 - Mean lenght of the CoP Path with eyes open (OE) and eyes closed (CE).

total length of the trajectory traveled by the Center of Pressure (CoP) (Figure 4).

Finally, the Romberg index also showed some improvements in the three different measurement times, albeit statistically insignificant.

\section{DISCUSSION}

Several studies have shown the effectiveness of ESWT on MPS.

Müller-Ehrenberg and Licht reported that the ESWT focused on MTPs alleviated pain in 3 months for $95 \%$ of 30 patients ( 800 pulses of energy level: $0.04-0.26$ $\mathrm{mJ} / \mathrm{mm} 2 ; 6 \mathrm{~Hz} ; 7$ treatments on average, 2 sessions per week) (Müller-Ehrenberg \& Licht, 2005).

In Jeon's study, the weekly focused ESWT (3 sessions of 1500 pulses with $0.10 \mathrm{~mJ} / \mathrm{mm} 2$ ) was as effective as the TENS and TrP injection for controlling the pain of the MP in the trapezius muscle on 30 patients.

In another study, the combination of ESWT and shoulder stabilization exercises was effective in reducing pain and restoring function in patients with MPS.

Several previous studies have investigated how ESWT impacts MPS.

Considering the hypothesis of the energy crisis and the mechanotransduction effect of rESWT in other diseases, rESWT in MPS probably facilitates perfusion, helps the reformation of blood vessels (angiogenesis effect), facilitates the recovery of connective tissue, provides adenosine triphosphate to the blood flow around the TrPs and alters the signaling of pain in the ischemic tissues caused by the influx of calcium when applied in depth on the injured regions. In addition, a recent animal study tested the hypothesis that ESWT's chronic pain relief is due to selective loss of nerve fibers in peripheral nerves (Hausdorf et al., 2008).

One study stated that ESWTs were involved in improving ROM of the shoulder joints and in recovering cervical functions, since the ESWT gun limits unwanted movements in joint exercises and provides stability (Cho et al., 2012).

Despite the extensive use of ESWT worldwide, studies of the dose relationship between the intensity and biological effects of ESWT remain inadequate.
Furthermore, to our knowledge, there have been only a few studies for MPS of this type.

Therefore, we decided to use low energy rESWT for patients with MPS because low energy rESWT usually has few side effects and good efficacy.

Previous studies have shown that high intensity energy stimulates more unmyelinated sensory nerve fibers, and therefore has a greater pain-reducing effect (Takahashi, 2006).

The histological reaction to ESWT is known to be dose dependent on the total energy delivered to the tissue.

Our study showed an improvement in statistical significance in pain sensation and biopostural data.

The functional improvement of the application of rESWT is probably caused by the pain reduction effect and by the stimulation of the fascial and muscular mechanoreceptors.

So functional improvement may follow pain reduction. In this way, the patients in our study showed pain reduction and functional and postural improvement. The likely side effects of rESWT include skin rash, pain, small hematoma, headache and so on. However, our patients had no major adverse effects.

The present study has several limitations. First, it has demographic limits (only a small number of subjects and limited age distribution).

Second, since it has been conducted for a short period of time, it has not been able to fully assess the longterm effects of low energy rESWT.

More precisely, although the effect of neuromodulation may be reflected in the results of the present study, the improvement of tissue perfusion in ischemic tissue through angiogenesis could not be reflected in such a short period of time.

Finally, the present study does not have a control group to rule out the placebo effect.

\section{CONCLUSIONS}

Both focused and radial extracorporeal shock waves are single acoustic pulses with a high initial positive peak pressure between 10 and $100 \mathrm{MPa}$ reached in less than $1 \mu \mathrm{s}$.

The amplitude of the positive pressure is followed by a low traction amplitude lasting a few microseconds which can generate cavitation (Angstman, N. B. et al 2015).

They are also characterized by a short life cycle of approximately $10-20 \mu$ s and a wide spectrum of frequencies.

The focused ESWs differ from the rESWs in the depth of penetration into the tissue, in some physical characteristics and in the technique to generate them (Ogden, J. A. 2001).

Therefore, the use of radial shock waves in problems related to myofascial pain and postural implications would be desirable.

Although our study has demonstrated a myofascial therapeutic path with improvements, the exact mechanism of the application of rESWT on functional improvement has not been studied exactly so far.

Therefore, future studies should be conducted over a longer period of time to investigate thoroughly the effects of this new therapeutic path. 


\section{REFERENCES}

1. Angstman, N. B., Kiessling, M. C., Frank, H. G., \& Schmitz, C. (2015). High interindividual variability in dosedependent reduction in speed of movement after exposing C. elegans to shock waves. Frontiers in behavioral neuroscience, 9, 12.

2. Aprile, I., Bordieri, C, Gilardi, A., Lainieri Milazzo, M., Russo, G., et al. (2013). Balance an.d walking involvement in facioscapulohumeral dystrophy: a pilot study on the effects of custom lower limb orthoses. Eur J Phys Rehabil Med., 49(2), 169-178.

3. Bellomo, R. G., Di Giulio, C., Giannuzzo, G., Irace, G., et al. (2018). Effects of manual somatic stimulation on the autonomic nervous system and posture. In Progress in Medical Research (pp. 97-109). Springer, Cham.

4. Barassi, G., Bellomo, R. G., Porreca, A., Giannuzzo, G., Giannandrea, N., et al. (2019). The use of adaptive neuro-stimulation for rebalancing posture and muscular tone in a soccer team. The Journal of sports medicine and physical fitness, 59(10), 1676-1683.

5. Bellizzi, M., Rizzo, G., Bellizzi, G., Ranieri, M., Fanelli, M., et al. (2013) Electronic baropodometry in patients affected by ocular torticollis. Strabismus, 19(1), 21-25.

6. Cho, Y. S., Park, S. J., Jang, S. H., Choi, Y. C., Lee, J. H., \& Kim, J. S. (2012). Effects of the combined treatment of extracorporeal shock wave therapy (ESWT) and stabilization exercises on pain and functions of patients with myofascial pain syndrome. Journal of Physical Therapy Science, 24(12), 1319-1323.

7. Frairia, R., \& Berta, L. (2011). Biological effects of extracorporeal shock waves on fibroblasts. A review. Muscles, ligaments and tendons journal, 1(4), 138.

8. Gerdesmeyer, L., Maier, M., Haake, M., \& Schmitz, C. (2002). Physical-technical principles of extracorporeal shockwave therapy (ESWT). Der Orthopade, 31(7), 610-617.

9. Hausdorf, J., Lemmens, M. A. M., Heck, K. D. W., Grolms, N., Korr, H., et al. (2008). Selective loss of unmyelinated nerve fibers after extracorporeal shockwave application to the musculoskeletal system. Neuroscience, 155(1), $138-144$

10. Horn, C., Mengele, K., Gerdesmeyer, L., Gradinger, R., \& Gollwitzer, H. (2009). The effect of antibacterial acting extracorporeal shockwaves on bacterial cell integrity. Medical Science Monitor, 15(12), BR364-BR369.

11. Kuan, T. S., Hong, C. Z., Chen, J. T., Chen, S. M., \& Chien, C. H. (2007). The spinal cord connections of the myofascial trigger spots*,**. European Journal of Pain, 11(6), 624-634.

12. Kuo, Y. R., Wang, C. T., Wang, F. S., Chiang, Y. C., \& Wang, C. J.(2009). Extracorporeal shock*wave therapy enhanced wound healing via increasing topical blood perfusion and tissue regeneration in a rat model of STZ*induced diabetes. Wound Repair and Regeneration, 17(4), 522-530.

13. Lee, S. Y., Cheng, B., \& Grimmer-Somers, K.(2011). The midterm effectiveness of extracorporeal shockwave therapy in the management of chronic calcific shoulder tendinitis. Journal of shoulder and elbow surgery, 20(5), 845-854.

14. Müller-Ehrenberg, H., \& Licht, G. (2005). Diagnosis and therapy of myofascial pain syndrome with focused shock waves (ESWT). Medizinisch-Orthopädische Technik, 5, 1-6.

15. Ogden, J. A., Tóth-Kischkat, A., \& Schultheiss, R.(2001). Principles of shock wave therapy. Clinical Orthopaedics and Related Research ${ }^{\circledR}, 387,8-17$.

16. Ramon, S., Gleitz, M., Hernandez, L., \& Romero, L. D. Hernandez, L., \& Romero, L. D. (2015). Update on the efficacy of extracorporeal shockwave treatment for myofascial pain syndrome and fibromyalgia. International Journal of Surgery, 24, 201-206.

17. Schmitz, C., Császár, N. B., Milz, S., Schieker, M., Maffulli, N., Rompe, J. D., \& Furia, J. P. (2015). Efficacy and safety of extracorporeal shock wave therapy for orthopedic conditions: a systematic review on studies listed in the PEDro database. British medical bulletin, 116(1), 115.

18. Shah, J. P., Danoff, J. V., Desai, M. J., Parikh, S., Nakamura, L. Y., Phillips, T. M., \& Gerber, L. H. (2008). Biochemicals associated with pain and inflammation are elevated in sites near to and remote from active myofascial trigger points. Archives of physical medicine and rehabilitation, 89(1), 16-23.

19. Simons, D. G. (1996). Clinical and etiological update of myofascial pain from trigger points. Journal of musculoskeletal pain, 4(1-2), 93-122.

20. Takahashi, N., Ohtori, S., Saisu, T., Moriya, H., \& Wada, Y. (2006). Second application of low-energy shock waves has a cumulative effect on free nerve endings. Clinical Orthopaedics and Related Research $\mathbb{R}, 443,315$ 319.

21. Vetrano, M., d'Alessandro, F., Torrisi, M. R., Ferretti, A., Vulpiani, M. C., \& Visco, V. (2011). Extracorporeal shock wave therapy promotes cell proliferation and collagen synthesis of primary cultured human tenocytes. Knee surgery, sports traumatology, arthroscopy, 19(12), 2159-2168.

22. Wang, C. J. (2003). An overview of shock wave therapy in musculoskeletal disorders. Chang Gung medical journal, 26(4), 220-232.

23. Wang, C. J. (2012). Extracorporeal shockwave therapy in musculoskeletal disorders. Journal of orthopaedic surgery and research, 7(1), 11.

24. Yin, T. C., Wang, C. J., Yang, K. D., Wang, F. S., \& Sun, Y. C. (2011). Shockwaves enhance the osteogenetic gene expression in marrow stromal cells from hips with osteonecrosis. Chang Gung Med J, 34(4), 367-74. 